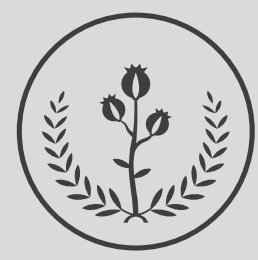

FUCS
Re

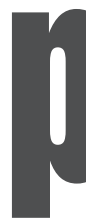

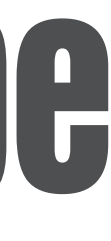

\title{
Observación del plan de vacunación del COVID-19 en Colombia
}

\section{Observation of the covid-19 vaccination plan in Colombia}

a Ingeniero. Magister en Gestión de la Tecnología Educativa, Especialista en Administración de la Informática Educativa. Docente de matemáticas e Investigador, Secretaría de Educación de Soacha, Cundinamarca.

\section{R E S U M E N}

Introducción: desde la identificación de los primeros casos de enfermedad por coronavirus 2019 (COVID-19) originada por el síndrome respiratorio agudo severo coronavirus 2 (SARS-COV-2) en diciembre 2019, la pandemia resultante ha dado lugar a más de 210 millones de casos confirmados y más de 4,4 millones de muertes en todo el mundo a 6 de abril 2021. Objetivo: mostrar el comportamiento del plan de vacunación contra el SARS-COV-2 entre el periodo comprendido del 17 de febrero al 17 de agosto 2021. Metodología: estudio transversal con fuente de información del plan de vacunación nacional contra el COVID-19 de la página web del Ministerio de Salud y Protección Social. Resultados: al 6 de agosto se evidenció el mayor número de aplicaciones (594.993 dosis), el 29 de junio el mayor número segunda dosis (235.048), el 15 de julio tuvo el mayor de monodosis (165.266) y el 9 de julio el de esquemas completos (337.354). Conclusión: es de vital importancia realizar un seguimiento al proceso de vacunación para entender su eficacia, la posible disminución de la respuesta inmune con el tiempo y los futuros efectos adversos.

Palabras clave: COVID-19, SARS-COV-2, pandemia, vacunas.

(C) 2021 Fundación Universitaria de Ciencias de la Salud - FUCS. Este es un artículo Open Access bajo la licencia CC BY-NC-ND (http://creativecommons.org/licenses/by-nc-nd/4.0/).

\section{INFORMACIÓN DEL ARTÍCULO}

Historia del artículo:

Fecha recibido: agosto 20 de 2021 Fecha aceptado: septiembre 24 de 2021
Autor para correspondencia: Ing. Jorge Enrique Díaz Pinzón jediazp@unal.edu.co
DOI

10.31260/RepertMedCir.01217372.1273 


\section{A B S T R ACT}

Introduction: since the first cases of coronavirus 2019 disease (COVID-19), caused by severe acute respiratory syndrome coronavirus 2 (SARS-COV-2), were identified on December 2019, the resulting pandemic has led to 210 million confirmed cases and more than 4.4 million deaths around the globe as of April 6 2021. Objective: to measure the SARS-COV-2 vaccination plan performance in the period from February 17 to August 17 2021. Methodology: a cross-sectional study using the information released in the Ministry of Health and Social Protection webpage based on the national vaccination plan. Results: the highest number of doses (594.993 doses), was administered on August 6, the highest number of second doses was administered on June 29 (235.048), the highest number (165.266) of single dose regimens was administered on July 15 and the most completed vaccination schedules (337.354) were achieved on July 9. Conclusion: monitoring the vaccination process is essential to gain understanding on the effectiveness, a possible drop of immune response over time, and the future effects of these vaccines.

Key words: COVID-19, SARS-COV-2, pandemic, vaccines.

(C) 2021 Fundación Universitaria de Ciencias de la Salud - FUCS. This is an open access article under the CC BY-NC-ND license (http://creativecommons.org/licenses/by-nc-nd/4.0/).

\section{INTRODUCCIÓN}

El SARS (síndrome respiratorio agudo grave) es el estadio grave de la COVID-19 originado por un daño masivo alveolar y una falla respiratoria progresiva ocasionado por el SARSCOV-2 (coronavirus 2 del SARS). ${ }^{1}$

La rápida tarea (vacunas) contra el COVID-19 ha obligado hacer uso de la proteómica para buscar antígenos exclusivos del patógeno en la proteína S. Gracias a la bioinformática se han podido reconocer 933 pentapéptidos ausentes en el proteoma humano, de los cuales 107 péptidos se ubican alrededor de la proteína $\mathrm{S}$ y de éstos 66 péptidos son más inmunógenos y se pueden usar en la producción de una vacuna. $^{2}$ La Organización Mundial de la Salud (OMS) cuenta con 52 alternativas de candidatos a vacunas entre plataformas basadas en proteínas ARN, ADN, vectores no replicantes, replicantes, virus inactivados, atenuados y partículas tipo virus. De todos estos prospectos de vacuna solo las constituidas por ARN y por vector no replicante han iniciado estudios de seguridad en humanos., ${ }^{3,4}$

Para lograr una reducción continuada de los casos de infección ${ }^{5}$ se necesitan contramedidas múltiples medidas incluido el distanciamiento, las pruebas y el rastreo, más aún a la luz de la reciente aparición de nuevas variantes del SARS-COV-2 ${ }^{6}$, como B.1.1.7 y B.1.351, que según informaciones tienen mayor transmisibilidad ${ }^{7,8}$ y podrían causar una enfermedad más grave ${ }^{9}$ en comparación con la cepa original. No se espera que la vacunación por sí sola pueda contrarrestar la propagación de la infección, por lo tanto, es necesario regular una campaña de vacunación planificada. ${ }^{10,11}$

El objetivo de esta investigación es mostrar el comportamiento del plan de vacunación contra el SARSCOV-2, en el periodo comprendido entre el 17 de febrero al 17 de agosto 2021.

\section{METODOLOGÍA}

La presente investigación se efectuó bajo un estudio transversal, la fuente de información fue adquirió fue la página web del Ministerio de Salud y Protección Social. ${ }^{12}$ Se tomó el período completo (17 de febrero 2021 a 17 de agosto 2021), para analizar la evolución de casos diarios de primera dosis, segunda, monodosis y esquemas completos de vacunación para SARS-COV-2. Asimismo, se manejaron medios matemáticos y estadísticos cotidianos para valorar los resultados de modo concluyente. ${ }^{13-15}$

\section{RESULTA DOS}

En la figura 1 se aprecian los casos acumulados de vacunación contra SARS-COV-2 entre el 17 de febrero a 17 de agosto 2021, con las tendencias acumuladas para el total de dosis aplicadas, primera y segunda dosis, monodosis y esquemas completos a 17 de agosto 2021 (182 días desde el inicio del plan de vacunación en Colombia). El total de dosis aplicadas fue $32^{\prime} 192.874$, primera dosis $18^{\prime} 312.186$, segunda dosis 1'1'214.334, monodosis 2'666.354 y esquemas completos 13'880.688.

En la figura 2 se aprecian los casos diarios de vacunación contra SARS-COV-2 entre el 17 de febrero a 17 de agosto 2021. Se representa en ella las tendencias diarias para dosis aplicadas, primera y segunda dosis, monodosis y esquemas completos. El 6 de agosto se evidenció el mayor número de aplicaciones diarias (594.993) dosis, el 29 de junio el mayor número de segunda dosis (235.048), el 15 de julio el mayor de monodosis 165.266) y el 9 de julio el de esquemas completos (337.354). 


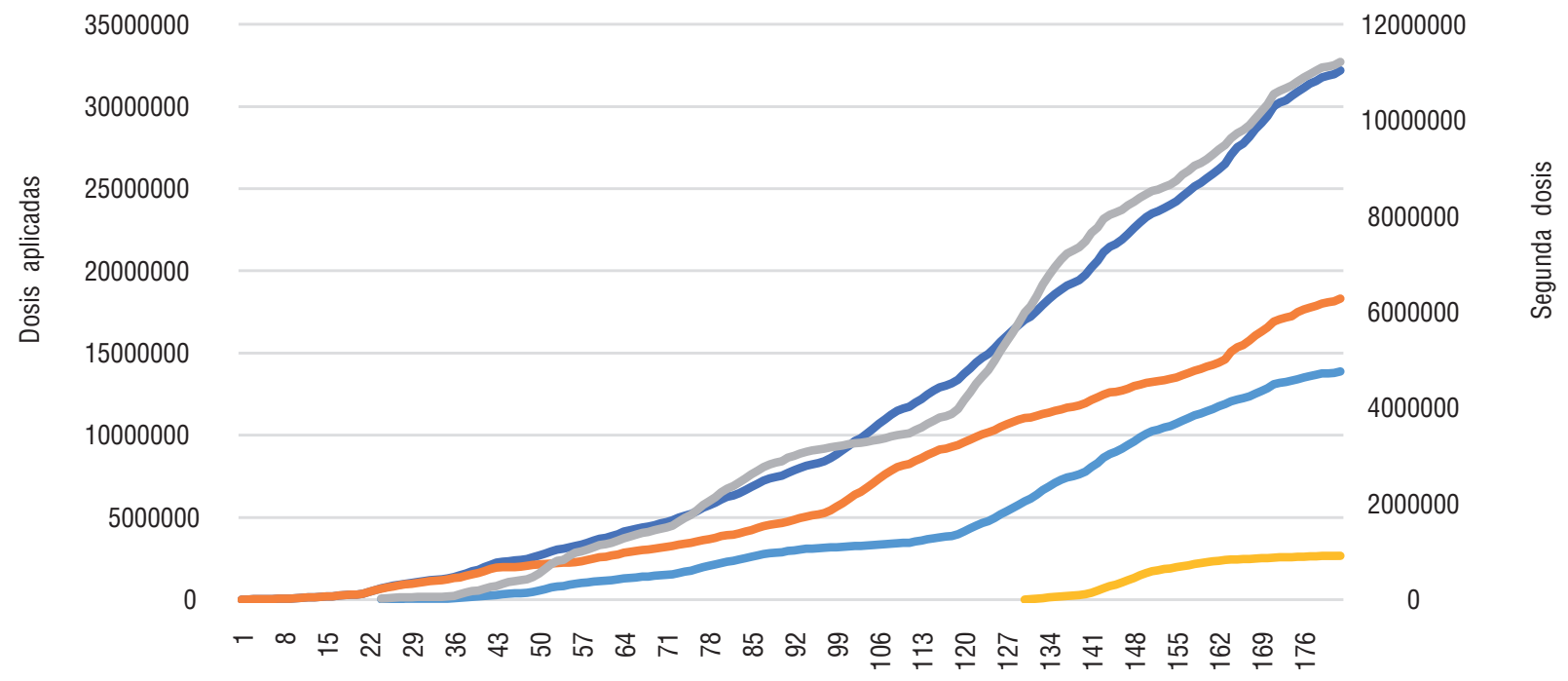

Días

Dosis aplicadas

Primera dosis

Monodosis

Esquema completo

Segunda dosis

Figura 1. Vacunación acumulada contra SARS-CoV-2. Fuente: el autor.

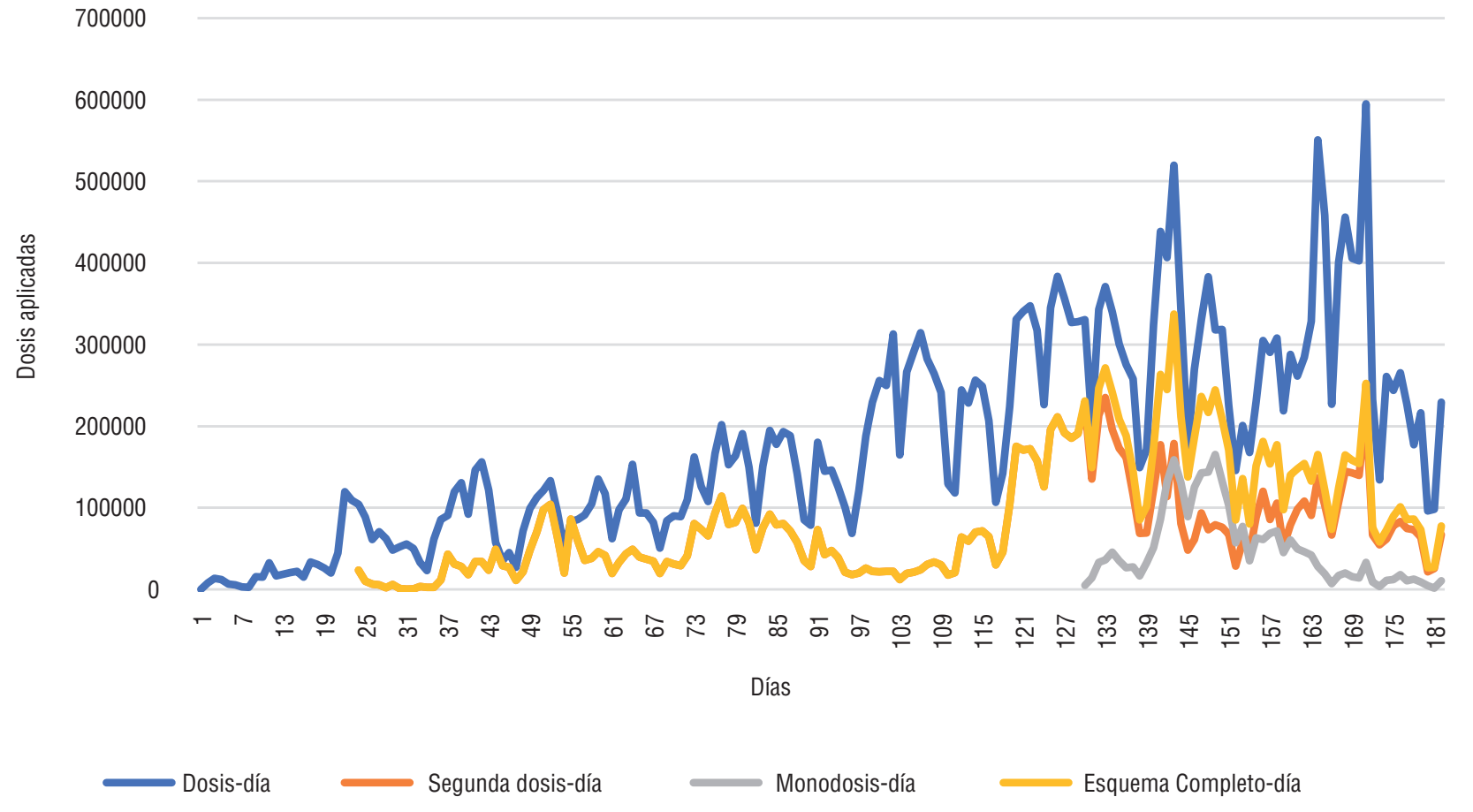

Figura 2. Diagrama de correlación por contagio y vacunación. Fuente: el autor. 


\section{CONCLUSIONES}

Es de vital importancia realizar un seguimiento al proceso de vacunación para entender su eficacia, la posible disminución de la respuesta inmune con el tiempo y los posibles efectos adversos. También es fundamental hacer el seguimiento a las mutaciones del virus que se presenten en el país, que afecten la inmunidad proporcionada por las vacunas. Hay que ampliar la vacunación a los menores entre 12 y 17 años con o sin comorbilidades, para que se produzcan efectos muy determinantes en la reducción de la mortalidad y del contagio por SARS-COV-2.

\section{CONFLICTO DE INTERESES}

El autor declara no tener ningún tipo de conflicto de interés.

\section{REFEREN CIAS}

1. Xu Z, Shi L, Wang, Zhang J, Huang L, Zhang C, et al. Pathological findings of COVID-19 associated with acute respiratory distress syndrome. Lancet Respir Med. 2020;8(4):420-422.. doi: 10.1016/ S2213-2600(20)30076-X.

2. Lucchese G. Epitopes for a 2019-nCoV vaccine. Cell Mol Immunol. 2020;17(5):539-540. doi: 10.1038/s41423-020-0377-z

3. Cohen J. Vaccine designers take first shots at COVID-19. Science. 2020;368(6486):14-16. doi: 10.1126/science.368.6486.14.

4. Lurie N, Saville M, Hatchett R, Halton J. Developing Covid-19 Vaccines at Pandemic Speed. N Engl J Med. 2020;382(21):19691973. doi: 10.1056/NEJMp2005630.

5. Priesemann V, Brinkmann MM, Ciesek S, Cuschieri S, et al. Calling for pan-European commitment for rapid and sustained reduction in SARS-CoV-2 infections. Lancet. 2021;397(10269):92-93. doi: 10.1016/S0140-6736(20)32625-8

6. Priesemann V, Balling R, Brinkmann MM, Ciesek S, Czypionka T, et al. An action plan for pan-European defence against new SARSCoV-2 variants. Lancet. 2021;397(10273):469-470. doi: 10.1016/ S0140-6736(21)00150-1
7. Davies NG, Abbott S, Barnard RC, Jarvis CI, Kucharski AJ, et al. Estimated transmissibility and impact of SARS-CoV-2 lineage B.1.1.7 in England. Science. 2021 Apr 9;372(6538):eabg3055. doi: 10.1126/science.abg 3055

8. Abbott S, Funk S, CMMID COVID-19 Working Group. Local area reproduction numbers and S-gene target failure [Internet]. 2021 [citado 19 de abril de 2021]. Disponible en: https://cmmid.github. io/topics/covid19/local-r-sgtf.html

9. Horby P, Huntley C, Davies N, Edmunds J, et al. NERVTAG paper on COVID-19 variant of concern B.1.1.7 [Internet]. Department of Health and Social Care; 2021 [citado 19 de abril de 2021]. Disponible en: https://www.gov.uk/government/publications/ nervtag-paper-on-covid-19-variant-of-concern-b117

10. Bubar KM, Reinholt K, Kissler SM, Lipsitch M, et al. Modelinformed COVID-19 vaccine prioritization strategies by age and serostatus. Science. 2021;371(6532):916-921. doi: 10.1126/science. abe6959

11. Ramos AM, Vela-Pérez M, Ferrández MR, Kubik AB, Ivorra, B. Modeling the impact of SARS-CoV-2 variants and vaccines on the spread of COVID-19. Commun Nonlinear Sci Numer Simul. 2021;102:105937. doi: 10.1016/j.cnsns.2021.105937

12. Ministerio de Salud y protección Social. Vacunación contra COVID-19 [Internet]. 2021 [citado 19 de abril de 2021]. Disponible en: https://www.minsalud.gov.co/salud/publica/Vacunacion/ Paginas/Vacunacion-covid-19.aspx

13. Díaz Pinzón JE. Análisis de los resultados del contagio del COVID-19 respecto a su distribución geográfica en Colombia. Repert Med Cir. 2020;29(Núm. Supl.1):60-64. https://doi. org/10.31260/RepertMedCir.01217372.1082

14. Díaz Pinzón JE. Perspectiva del tiempo para alcanzar la inmunidad de rebaño para COVID-19 a nivel mundial. Repert Med Cir. 2021;30(Núm. Supl.1):61-66. https://doi.org/10.31260/ RepertMedCir.01217372.1245

15. Díaz Pinzón, J. E. (2021). Proyección del tiempo para alcanzar la inmunidad de rebaño para COVID-19 en Bogotá. Repert Med Cir. 2021;30(Núm. Supl.1):79-83. https://doi.org/10.31260/ RepertMedCir.01217372.1252 